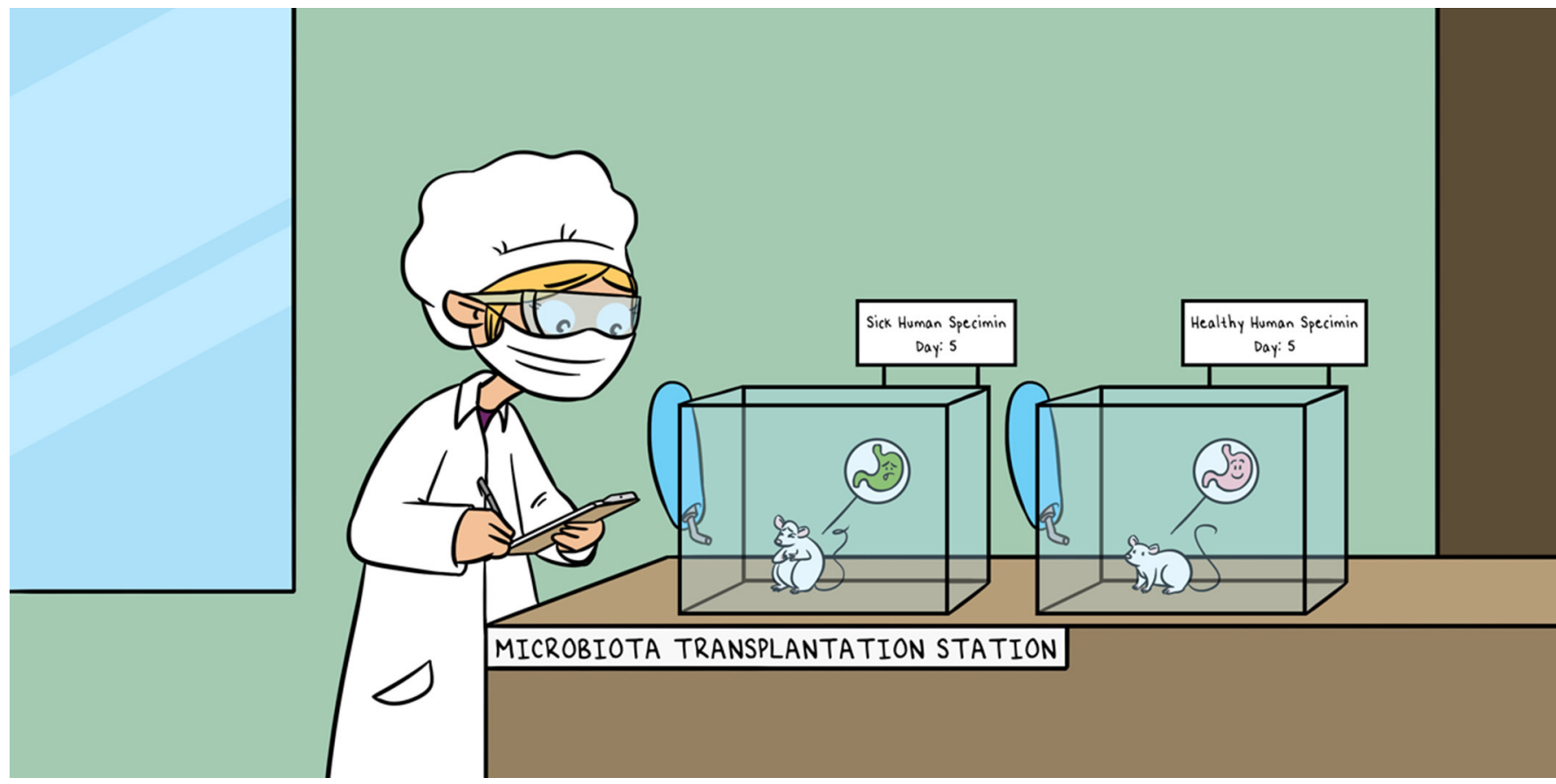

\title{
HOW THE GUT MICROBIOTA INFLUENCES OUR HEALTH AND HOW WE CAN INFLUENCE IT
}

\section{Markus Arnoldini ${ }^{1 *}$, Verena Lentsch ${ }^{1}$, Daniela Latorre ${ }^{2}$, Giorgia Greter ${ }^{1}$, Emma Slack $^{1}$, Ambra Giorgetti ${ }^{1}$, Alessandro Groppo ${ }^{3}$ and Lilian Kha ${ }^{3}$}

${ }^{1}$ Department of Health Science and Technology, Institute of Food, Nutrition and Health, ETH Zürich, Zürich, Switzerland

${ }^{2}$ Departement of Biology, Institute of Microbiology, ETH Zürich, Zürich, Switzerland

${ }^{3}$ ETH Zürich, Zürich, Switzerland

YOUNG REVIEWERS:

AISLING

AGE: 9

MRIDULA

AGE: 15

\section{MICROBES}

All living things that are not visible by the naked eye are called microbes. They are found in most places on earth, and perform important functions in the environment and for our health, but some microbes make us sick.
The microbes in the human gut, also known as the gut microbiota, are known to play a part in how we feel and whether we are healthy or sick. How the microbiota regulates health is still unknown in most cases, and medical treatments to manipulate the microbiota to improve people's health are only just starting to be used. In this article, we explain how the gut microbiota is currently studied, what we already understand about how the gut microbiota influences health, and ways that are already used, or are being investigated, to influence the microbiota to improve health.

\section{THE GUT MICROBIOTA: NUTS AND BOLTS}

They are in you, they are on you, they are a part of you ... they are microbes! Microbes are organisms so small that we cannot see them with our eyes. On and inside our bodies, we have roughly 
Figure 1

The gut

microbiota changes as we age. (A) In

newborns, very few

microbes are present in the gut. As a child

grows older, he or she picks up microbes from other people or the environment, which form the gut microbiota. (B,C) The gut microbiota changes and gets more diverse over time. The exact mix of microbes in every person is unique, and therefore everyone's microbiota looks different.

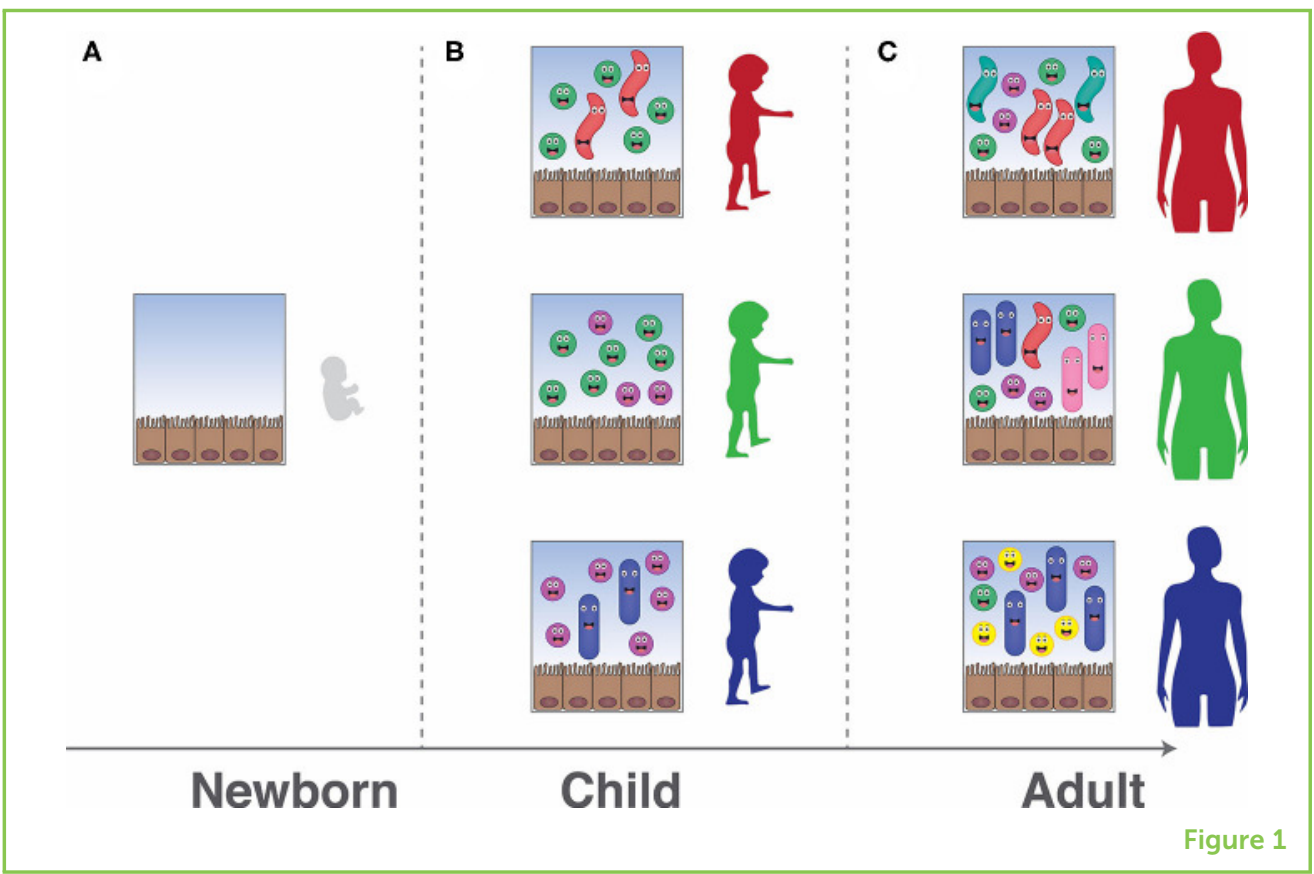

as many microbes as human cells. Microbes have been colonizing humans for millions of years, and we have evolved together. During this long association, we became dependent on each other: we offer the microbes food and a place to live, and in turn they work for us. We are only beginning to understand the many effects microbes have on us, but there are some surprising examples: they help us digest food, train our immune systems, protect us from infections, regulate our appetites, and even influence whether we feel happy or sad. Most of the microbes in our bodies live in the gut, which is one of the largest interfaces between you-the host-and the microbes. We call the entire collection of microbes living in the gut the gut microbiota.

The microbes in the gut are not all the same. So far, researchers have identified more than 2,000 different species of microbes that live in or on humans. Everyone differs in the number and abundance of species in the gut microbiota, which makes the microbiota of each person unique.

Where does the gut microbiota come from? There are few microbes living in or on babies before they are born. At birth, human babies meet some microbial friends for the first time, and they continue picking up new microbes as they grow older. The microbiota of children looks different from that of adults, and the number of species increases steadily until adulthood (Figure 1). Even in adults, the composition of the microbiota can still change it and depends on our genes, the environment we live in, and the foods we eat. 


\section{WHAT IS A HEALTHY GUT MICROBIOTA?}

We know that a healthy gut microbiota is important. But what is a healthy microbiota? This is an extremely difficult question to answer, even though many scientists are working hard on solving it. The number of different species and the relative abundances of these species vary between people and even within the same person over time. In addition, changes in the bodily environment can turn some microbiota species from harmless inhabitants into dangerous invaders. As a result, every time something changes in the gut, hundreds of other things change as well, which makes it extremely difficult for scientists to understand which of these changes is actually important.

How do scientists study the function of such a complex system? One way is to use mice that are raised in exceptionally clean conditions, so that they do not have any microbiota, but they are still healthy. Scientists give these mice some of the gut microbiota isolated from either sick or healthy humans. If only the mouse given the "sick" microbiota develops the disease, we know the disease is due to the microbiota, because it is the only difference between the mice. This is important to know, but in most cases, we still do not understand how this happens.

\section{HOW THE GUT MICROBIOTA CAN KEEP US HEALTHY}

In some cases, we have explanations for how the microbiota affects us. One such case is its interaction with the immune system. The immune system in the gut needs to prevent dangerous microbes from causing disease, but it should not over-react to the normal microbiota. The immune system achieves this state by producing a balance of activating and de-activating signals. If the balance is disturbed and mostly activating signals are produced, disease can result. Scientists have discovered that a waste product released by the microbiota, called butyrate, can instruct the immune system to make more deactivating signals. The microbiota thus helps keep the balance and prevents the immune system from overreacting [1].

A second case in which we have some understanding of how the microbiota affects us is the role of the microbiota in preventing gut infections. If you eat food or drink water containing microbes that can make you sick (Salmonella is one such microbe that you might have heard of), in many cases you actually stay healthy. This is often due to your gut microbiota, which can directly fight the invaders by producing substances that can harm them, by stimulating your immune system to increase your resistance to harmful bacteria, and by consuming all the available food so nothing is left for the invaders [2].

A third case is the role of the microbiota in harvesting energy from our food. Have you heard the term dietary fiber? Dietary fiber is abundant 
in fruits and vegetables, and it contains a lot of energy. But this energy cannot be used by our bodies because we do not have the machinery to extract it. However, the gut microbiota has this machinery, and the microbes can digest dietary fiber, releasing some of the energy for us to use. In this way, your microbiota produces $10 \%$ of the total energy that you use every day [3]. This is an important factor for survival if there is not enough food, but if there is too much food around, this process can potentially contribute to people becoming overweight.

\section{WAYS TO MANIPULATE THE GUT MICROBIOTA}

A healthy gut microbiota is important for some of the main functions of our bodies. But even if our microbiota is healthy, events like the invasion of dangerous bacteria or the use of antibiotics can dramatically change it. In some cases, these changes to the gut microbiota can cause diseases.

In the last decades, ways to help us keep a healthy microbiota or to manipulate the microbiota's composition have been discovered. There are also interventions that help to promote specific beneficial microbes and to inhibit ones known to be dangerous. Here are three such interventions (also see Figure 2).

\section{Prebiotics}

Different microbes have different food preferences. One way of supporting beneficial microbes is to help them grow by providing them

\section{PREBIOTICS}

Components in food that specifically feed beneficial members of the gut microbiota, to promote them over other species.

FECAL MICROBIAL TRANSPLANT

The transfer of the gut microbiota in the feces of a healthy person to a person with a disturbed gut microbiota. This technique is currently used for treating recurrent $C$. difficile infections. with the foods they like best [4]. These preferred dietary substances are called prebiotics. For example, some species of beneficial bacteria like to eat a certain kind of dietary fiber. One way to think about this would be as a car race, with different cars needing different kinds of fuel. Eating prebiotics is like giving fuel only to the cars you want to win (the beneficial bacteria), while not fueling the others.

\section{Fecal Transplantation}

Another way to change the microbiota is the transfer of the gut microbiota from a healthy person (the donor) to a sick one (the recipient). This is called a fecal microbial transplant, and you can think about it like replacing a soccer team that does not play well together with a different team. A fecal microbial transplant is done by taking the donor's gut microbiota from their feces, and transferring it into the recipient's gut, either by swallowing a pill or via a tube through the nose or the rectum. Does not sound very appealing to you? A very understandable reaction! However, for one disease, fecal microbial transplants seem to work extremely well: recurrent infections with Clostridioides difficile, a microbe that can thrive if the gut microbiota is disturbed, causing a very serious disease. C. difficile can be killed by antibiotics, but antibiotic resistance is common, and often the disease comes back when the patient stops taking the antibiotics. 
Figure 2

Manipulating the gut microbiota to improve health. (A) Prebiotics are components of food that can specifically feed desired microbes. The beneficial microbes will then increase in the gut, which will change the microbiota, hopefully eliminating the undesired microbes. (B) Fecal microbiota transplants put a healthy person's gut microbiota into a sick person's gut. These transplants can work well for treating infections with a dangerous bacterium called C. difficile. (C) Vaccination against a single type of microbe in the microbiota can train the immune system to make antibodies against this microbe, which helps the body to flush out the undesired microbes.

\section{ORAL VACCINATION}

Training the immune system to react against specific microbes by swallowing the killed microbe, or parts of the microbe.

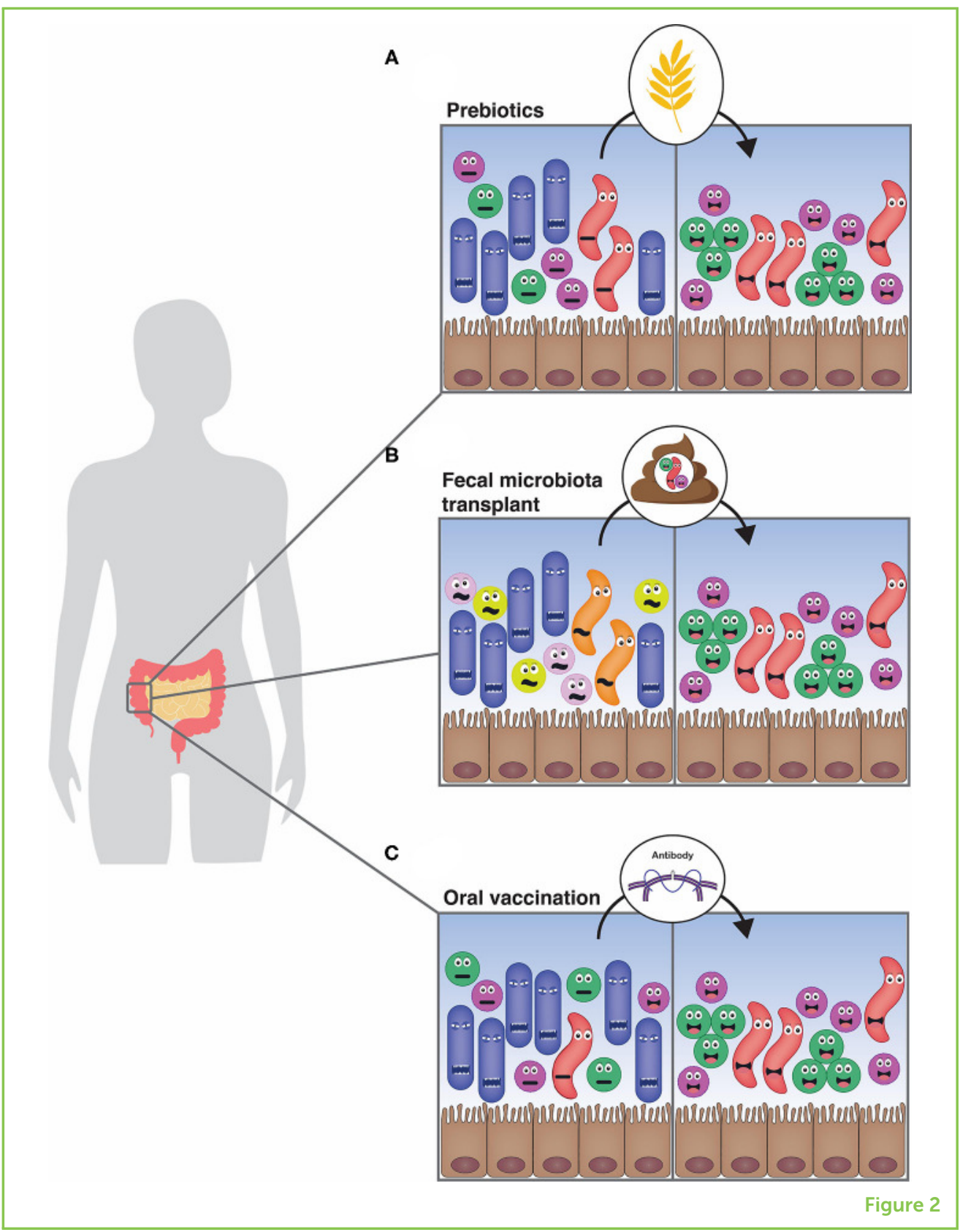

Fecal microbial transplants cure $80-90 \%$ of people suffering from this disease and they are generally believed to be safe. However, fecal microbial transplantation often influences the recipient's gut microbiota in an unpredictable way.

\section{Precision Microbiota Engineering}

Current research focuses on more targeted changes to the microbiota, to avoid some of the unpredictability of fecal microbial transplants. One possibility is oral vaccination against specific unwanted microbes. Like most other vaccines, oral vaccination tells the immune system to make proteins called antibodies that stick to the targeted microbes. These antibodies glue these microbes together into large clumps, which are easier to flush out of the gut [5]. To help this process, we 


\section{NICHE}

\section{COMPETITORS}

Members of a biological community that need the same resources (live in the same ecological niche) and are in direct competition for them can use another trick: a microbe that is very similar to the one targeted by the vaccine, but not harmful, can be given with the vaccine. This microbe is called a niche competitor. The niche competitor makes life even harder for the problematic microbe because it competes with the problematic microbe for food and living space. Imagine this like playing musical chairs: the immune system keeps the targeted microbes at distance and whoever is faster and better adapted can take their place. This strategy shows promising results, and research is underway to make it broadly usable.

\section{TAKE-HOME MESSAGE}

We are only beginning to understand the ways in which the gut microbiota influences your health and well-being, but we already know it has a strong effect and that there are smart ways to influence it. Understanding the role of the gut microbiota is a highly active area of research. As some groups around the world work to define what characteristics make a microbiota "healthy" or "diseased," others are continuing to develop techniques to make precise changes in the microbiota. This research will shed more light on the complex relationships between humans and microbes, and will lead to better ways for improving the health and quality of life for many people.

\section{REFERENCES}

1. Furusawa, Y., Yuuki, O., Fukuda, S., Endo, T. A., Nakato, G., Takahashi, D., et al. 2013. Commensal microbe-derived butyrate induces the differentiation of colonic regulatory T cells. Nature 504:446-50. doi: 10.1038/nature12721

2. Stecher, B., and Hardt, W. D. 2011. Mechanisms controlling pathogen colonization of the gut. Curr. Opin. Microbiol. 14:82-91. doi: 10.1016/ j.mib.2010.10.003

3. McNeil, N. I., Cummings, J. H., and James, W. P. 1978. Short chain fatty acid absorption by the human large intestine. Gut 19:819-22. doi: 10.1136/ gut.19.9.819

4. Shepherd, E. S., DeLoache, W. C., Pruss, K. M., Whitaker, W. R., and Sonnenburg, J. L. 2018. An exclusive metabolic niche enables strain engraftment in the gut microbiota. Nature 557:434-8. doi: 10.1038/s41586-018-0092-4

5. Moor, K., Diard, M., Sellin, M. E., Felmy, B., Wotzka, S. Y., Toska, A., et al. 2017. High-avidity IgA protects the intestine by enchaining growing bacteria. Nature 544:498-502. doi: 10.1038/nature22058

SUBMITTED: 26 June 2020; ACCEPTED: 05 February 2021; PUBLISHED ONLINE: 10 March 2021.

EDITED BY: Suzanne Phelan, California Polytechnic State University, CA, United States

CITATION: Arnoldini M, Lentsch V, Latorre D, Greter G, Slack E, Giorgetti A, Groppo $A$ and Kha $L$ (2021) How the Gut Microbiota Influences Our Health and How We 
Can Influence It. Front. Young Minds 9:576428. doi: 10.3389/frym.2021.576428

CONFLICT OF INTEREST: The authors declare that the research was conducted in the absence of any commercial or financial relationships that could be construed as a potential conflict of interest.

COPYRIGHT @ 2021 Arnoldini, Lentsch, Latorre, Greter, Slack, Giorgetti, Groppo and Kha. This is an open-access article distributed under the terms of the Creative Commons Attribution License (CC BY). The use, distribution or reproduction in other forums is permitted, provided the original author(s) and the copyright owner(s) are credited and that the original publication in this journal is cited, in accordance with accepted academic practice. No use, distribution or reproduction is permitted which does not comply with these terms.

\section{YOUNG REVIEWERS}
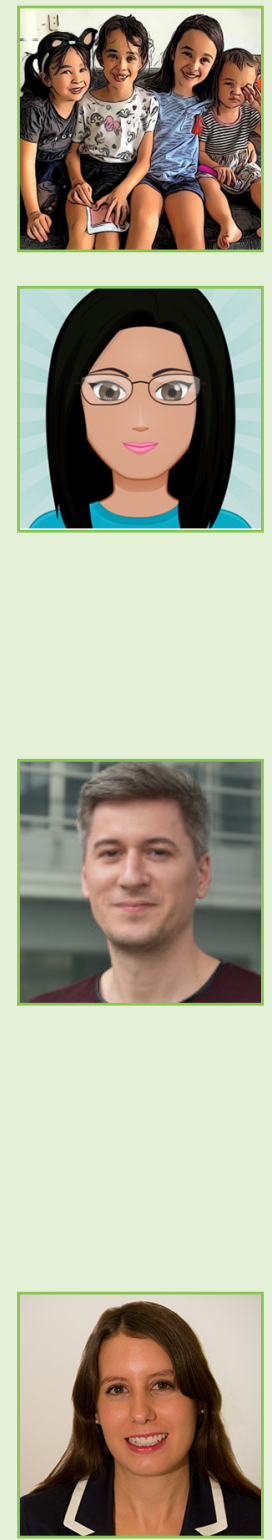

\section{AISLING, AGE: 9}

$\mathrm{Hi}$, I am Aisling! I love writing creative stories and I love school and wish it was longer. My favorite vegetables are broccoli and bok choy, and my favorite food is ddebokki and ramen!

\section{MRIDULA, AGE: 15}

$\mathrm{Hi}, \mathrm{I}$ am Mridula. Science and English have always been among my favorites in school. That is why I was highly interested when I found out about the Frontiers Young Minds community. I am excited to hopefully provide some helpful feedback to authors, and simultaneously learn about the powerful happening in science around the world.

\section{AUTHORS}

\section{MARKUS ARNOLDINI}

These days, everyone knows that we have tiny microorganisms in and on our bodies. They are important for our health, but sometimes also make us sick. I am investigating how our bodies and behaviors select which microorganisms live inside us and how they interact with each other. Are the "good bugs" always good, or can they sometimes turn against us and cause diseases? I do this research at ETH Zürich in Switzerland. In addition to doing experiments and analyzing the data, an important part of being a scientist is making sure that our findings reach people, so that scientific facts can be used as the basis for making informed decisions. *markus.arnoldini@ ahest.ethz.ch

\section{VERENA LENTSCH}

How can we make effective oral vaccines? What else do we need to change the gut microbiota in a targeted way? Can we use this approach to reduce antibiotic-resistant bacteria? These are the questions keeping me busy during my doctorate at ETH Zurich in Switzerland. In addition to the research in the lab, I also try to share my findings not only with fellow scientists but also with the public. If 

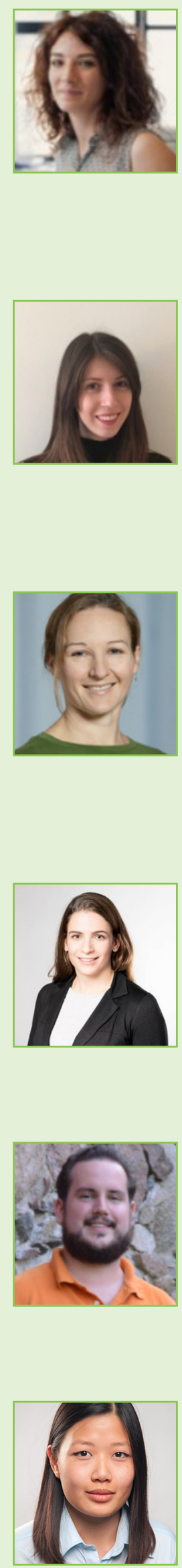

we do this effectively, we can help everyone to differentiate between real news and fake news.

\section{DANIELA LATORRE}

How does the immune system work in the context of infections? Which immune cell population are involved? How can we improve immune responses to prevent or better treat infections? I have been studying these aspects in healthy individuals and patients affected by immunodeficiencies or autoimmunity. I work at ETH Zurich, in Switzerland. I believe that spreading scientific knowledge to young people and the non-scientific community is part of our role as scientists, to help everyone understand science and to avoid the spreading of fake news.

\section{GIORGIA GRETER}

Did you know that you have a tiny ecosystem inside of your gut? All the members interact in a large network, which means that changing one thing inside of you changes everything around it. I am trying to answer some of the questions about how the bacteria in the gut are interconnected. I do my research in a laboratory at ETH in Zurich, Switzerland. One of the most important parts of my job is to communicate my research with the rest of the world, to help shape the public's general knowledge.

\section{EMMA SLACK}

Do you ever wonder about how your immune system works in your guts? How we keep the bad bugs out and keep the good bugs in? How our food affects our health? Can we make effective vaccines that we can swallow? Can we make people and farm animals healthier by changing their gut bacteria? These questions keep me and my research group very busy indeed. We work at ETH Zurich, in Switzerland. I also love to teach and communicate about science so that everyone, not just the scientists, can keep up to date with the amazing progress that is being made.

\section{AMBRA GIORGETTI}

I am a Ph.D. student at the laboratory of Human Nutrition at ETH Zurich. I investigate possible ways to improve the safety of nutritional supplements, specifically those containing iron. Iron supplements trigger the growth of some bad bacteria in our microbiota, which might lead to the development of some illnesses, such as diarrhea. In my projects, we try to support the good microorganisms of the microbiota by adding the food they like most-dietary fiber-to the iron formulations.

\section{ALESSANDRO GROPPO}

I am a master's student from Zurich, Switzerland, majoring in nutrition and health at the Department of Health Sciences and Technology at ETH Zurich. Since I became fascinated by the gastrointestinal tract and its relation to health and the microbiota, I dove deeper into this field by pursuing the class "Food, Microbiota, and Immunity: Debating the Evidence" and am now very keen to share my results and passion with others.

\section{LILIAN KHA}

I am a curious food-enthusiast, studying for a master's in food science at ETH Zurich. I have studied the importance of the gut microbiota and its involvement with us and 
our surroundings. This led to a fascination with this topic, which I want to share with others. 